\title{
Metodologie integrate per la conoscenza, la tutela e la rappresenta- zione dell'architettura militare storica. Sistemi costruttivi e percorsi voltati lungo i bastioni occidentali di Cagliari (Sardegna, Italia)
}

Integrated methodologies for the knowledge, representation and protection of historical military architecture. Construction systems and vaulted paths along the western bastions of Cagliari (Sardinia, Italy)

\section{Andrea Pirinu a, Vincenzo Bagnolob ${ }^{\text {, }}$, Raffaele Argiolas ${ }^{\text {c }}$, Marco Utzeri ${ }^{d}$ \\ Dipartimento di Ingegneria Civile, Ambientale e Architettura - Università degli Studi di Cagliari, Cagliari, Italy a apirinu@ unica.it; ${ }^{\mathrm{b}}$ vbagnolo@ unica.it; ${ }^{\mathrm{c}}$ raffaele.argiolas@ hotmail.com; ${ }^{\mathrm{d}}$ ing.marcoutzeri@ gmail.com}

\begin{abstract}
The integrated method is been applied in the meet point between the curtain of Santa Chiara and the curtain of de Cardona, in a limited area of the ancient walls of Cagliari (Sardinia, Italy). The sector consists of a bastion called "curtain of Santa Chiara" designed in 1575 and realized in the period 1575-1578 by the military engineer Giorgio Paleari and the "curtain of de Cardona", a military work commissioned by the Viceroy in the 1930s of the same century and interested by modification until the seventeenth century.

The archive documents offer a lot of informations on the presence of gunboats and vaulted passages designed and built in this area during this period. This source -accompanied by an architectural survey of the existing military work and the knowledge of the construction techniques used at the time and indicated in the military treaties- may direct a subsequent investigation with geophysical methods. To this aim, a first graphic representation of the study area in the sixteenth century is proposed.
\end{abstract}

Keywords: Military architecture, integrated digital survey, geophysical non-destructive survey, Cagliari.

\section{Introduzione}

L'indagine sul patrimonio storico urbano rappresenta la fase preliminare necessaria per l'identificazione dei migliori interventi e delle strategie di pianificazione. Una volta completata l'acquisizione e l'analisi della documentazione archivio, l'obiettivo principale da raggiungere è l'organizzazione di un database digitale che consenta di rappresentare attraverso modelli bidimensionali e tridimensionali la complessità del sistema studiato e le sue trasformazioni. Tale risultato può essere raggiunto attraverso un rilievo alla scala architettonica e urbana il quale, supportato dall'analisi storica e archivistica, favorisce una rilettura delle trasformazioni intercorse e la formulazione di ipotesi sulla presenza di antiche cortine, camminamenti e torri oggi inglobate all'interno del tessuto urbano.

L'indagine sui percorsi interni all'opera militare può inoltre avvalersi del contributo offerto dai metodi geofisici, già applicati con discreti risultati nel settore oggetto di studio (Pirinu, Balia, 2018). 
Il modello digitale diviene un valido strumento per lo studio e la comprensione dello stratificato paesaggio urbano del quartiere Castello a Cagliari e di un ambito specifico dei bastioni occidentali, edificati a partire dal Cinquecento a ridosso del perimetro medievale.

Tale settore è costituito dall'innesto della cortina di Santa Chiara, progettata e realizzata nel periodo 1575-1578 dall'ingegnere militare Giorgio Paleari, alla cortina del de Cardona, edificata negli anni '30 dello stesso secolo e interessata sino agli inizi del Seicento da modifiche e ammodernamenti documentati (Rassu, 2003).
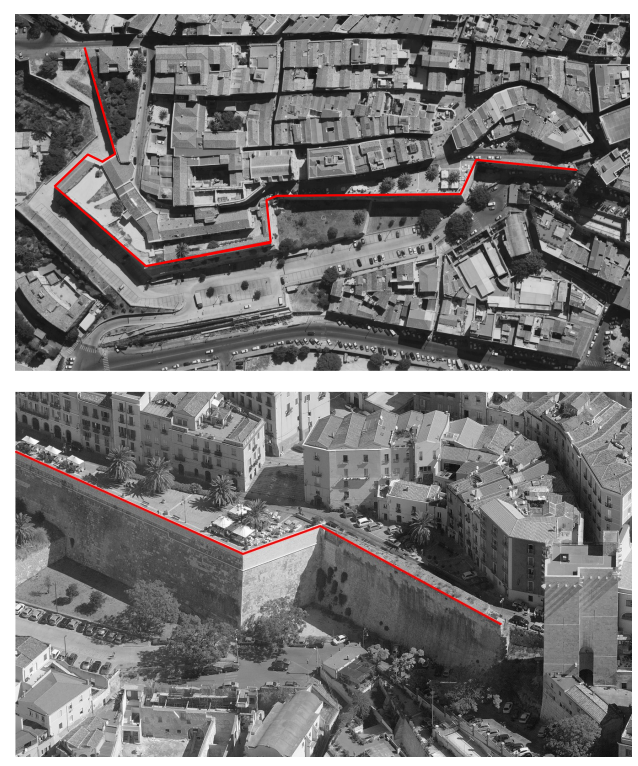

Fig. 1. Veduta ampia sui bastioni occidentali e dettaglio dell' area compresa tra la cortina di Santa Chiara e la torre dell'Elefante (RAS 2008).

\subsection{Forma e progetto della cortina di Santa Chiara a Cagliari}

Alcuni interessanti documenti descrivono il tratto della linea difensiva oggetto di studio.

A partire dal XVI secolo, disegni, relazioni tecniche e concessioni per l'uso delle mura della città forniscono informazioni utili per la conoscenza degli assetti medievali e delle trasformazioni "moderne" che hanno condotto all'attuale assetto. Tra questi la veduta pubblicata nel 1550 (Fig. 2) nella Cosmographia Universalis di
Munster, il progetto per le fortificazioni della città di Rocco Capellino datato 1552, i disegni del ticinese Giorgio Paleari risalenti al 1573, 1575 e 1578 e la rappresentazione settecentesca di Giuseppe Viana.

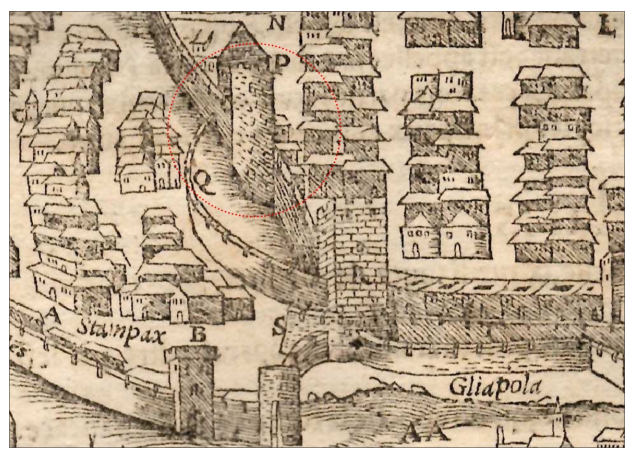

Fig. 2. Rappresentazione dell'area oggetto di studio nel 1550; la lettera "Q" indica la cortina del de Cardona. Si osserva la presenza di una torre circolare e di alcune case costruite vicino ad essa e citate in un documento datato 1537.

I primi due documenti, realizzati entrambi da tecnici militari (1552 e 1573), mostrano l'avanzamento del perimetro murato senza offrire ulteriori informazioni sul suo intorno, a differenza dei successivi disegni caratterizzati da una descrizione del tessuto edilizio presente a ridosso della linea di difesa tre-quantrocentesca.

Il progetto per la cortina di Santa Chiara, proposto nel 1575 (Fig. 3), consiste nella costruzione di una "cortina ripiegata" che fa avanzare il fronte per metà della sua lunghezza. Il progetto firmato "Georgio palearo fratino" è conforme a questa formulazione e prevede la demolizione delle otto case realizzate a ridosso del muro medievale, sebbene lo stesso ingegnere "sconsiglia di demolire case addossate all'antica cortina fra il baluardo di Santa Croce e Sant'Antonio e suggerisce di portare in fuori la muraglia per metà della lunghezza con un saliente munito di cannoniera, a incrociare i fuochi con il fianco ritirato del Balice" (Viganò 2004). L'indicazione "per metà della lunghezza", verificabile da un'analisi grafica del disegno (Pirinu, 2013), non trova tuttavia conferma nel confronto con la rappresentazione settecentesca eseguita dal Viana e la conformazione attuale; difatti la ripiegatura della 
nuova opera -nel primo disegno ad angolo retto ed "in appoggio" alla torre esistente- viene portata a compimento con una apertura tale da consentire la costruzione della casamatta, come mostra daltronte il successivo disegno del 1578 (Fig. 4). L'elaborato del 1575 evidenzia gli elementi chiave del progetto esaltando simmetrie rigorose, un perfetto allineamento tra faccia e fianco dei due bastioni esistenti (S. Croce e Balice), il ripiegamento dichiarato della nuova opera e la posizione delle nuove case da costruire tra la cortina di Santa Chiara e la torre dell'Elefante; gli edifici dovranno seguire il profilo delle mura medievali, queste ultime poggianti su una solida base rocciosa e distanziate dalla cortina del de Cardona da un terrapieno, come precisa il documento stesso.

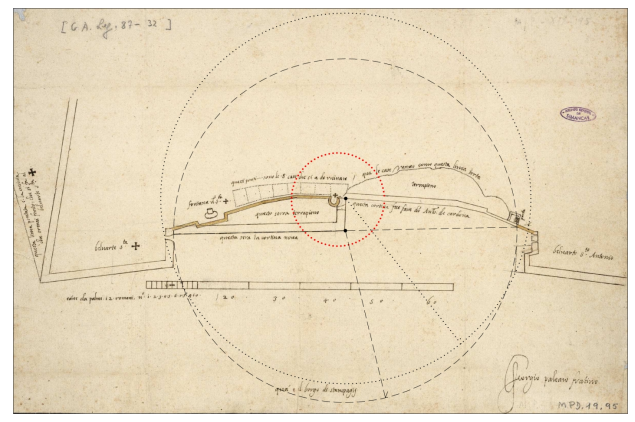

Fig. 3. Analisi grafica del progetto del 1575.

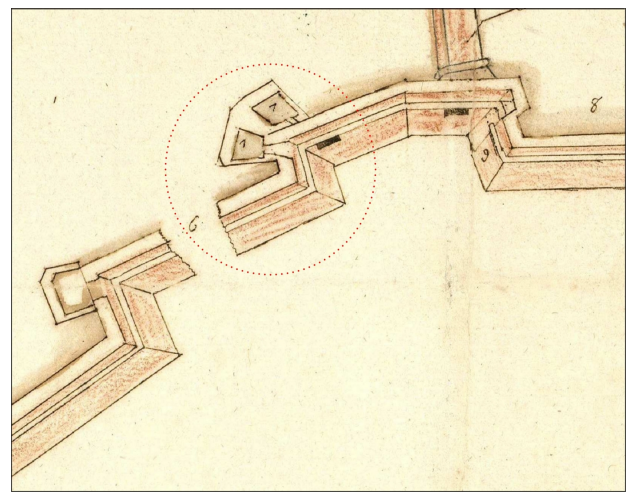

Fig. 4. La cortina di Santa Chiara nel 1578 con le sue due cannoniere indicate con il numero 7 (Archivo General de Simancas, MPD, 19,05, Spagna).

Il disegno del 1578 mostra le opere eseguite con la realizzazione del nuovo bastione dotato di due cannoniere, una delle quali in casamatta, il cui accesso è descritto da Giuseppe Viana nel documento del 1776 (Fig. 5).

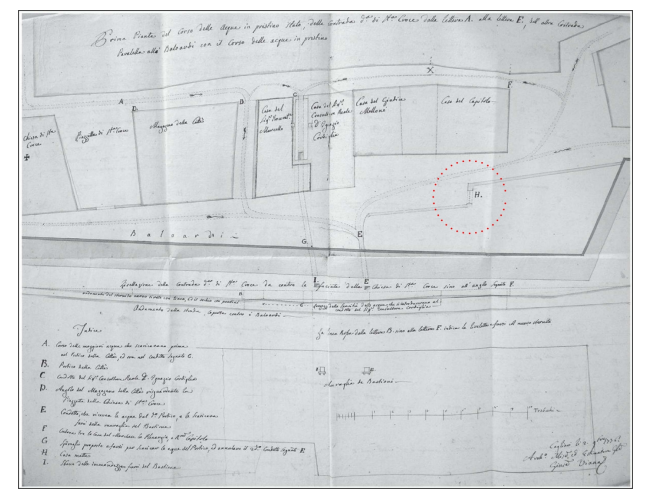

Fig. 5. Disegno di Giuseppe Viana che rappresenta la cortina di Santa Chiara, il contesto urbano e la "Casa matta" indicata con la lettera "H" (Archivio di Stato di Cagliari, Reale Udienza, Cause Civili. Disegno pubblicato in: Cadinu, Pirinu, Schirru, 2013, p. 540).

La ricerca archivistica offre anch'essa un interessante contributo all'indagine.

Alcuni documenti datati 1537 fanno riferimento all'uso, da parte di un commerciante ebreo di nome Gabriel Bonfill, di una torre (chiamata la Mordent) facente parte della mura del quartiere Castello, situata nel vicolo di Santa Croce o Orifany -a causa della presenza della vicina torre dell'Elefante- e confinante con il "mercato" del Balice. Anche se questa concessione non fu confermata, il mercante prese possesso dell'edificio comunque e ottenne di poter costruire nell'area adiacente alcune strutture alte come la torre, con la facoltà di poter costruire anche sopra le mura.

Luys Sabater, botiguer e sotaobrer della città di Cagliari, costruì invece una scala ed altre strutture abitative ornate di finestre che si affacciavano sulla via del Orifany, sempre vicine alla torre della Mordente e del passo di ronda (junct ab la muralla e marlets de la muralla), ma la concessione enfiteutica, a differenza di quella del Bonfill, fu sottoposta al benestare dei consiglieri cittadini e del viceré (Mele, 2019).

Questi riferimenti indicano chiaramente che la torre presente nel disegno del 1575 corrisponde a quella menzionata nei documenti e annessa ad 
una delle 8 case che saranno demolite, come previsto dal disegno del 1575 .

Tali informazioni, supportate da scale metriche in palmi romani e trabucchi (Schirru, 2017), unitamente alla conoscenza delle tecniche di progettazione e costruzione adottati dagli ingegneri militari (Figg. 6-9), guidano una prima ipotesi di ubicazione degli elementi del bastione celati al di sotto del livello stradale odierno (Milanese, 2008), scelta che non esclude una posizione della torre più prossima al punto d'incontro tra le due cortine.

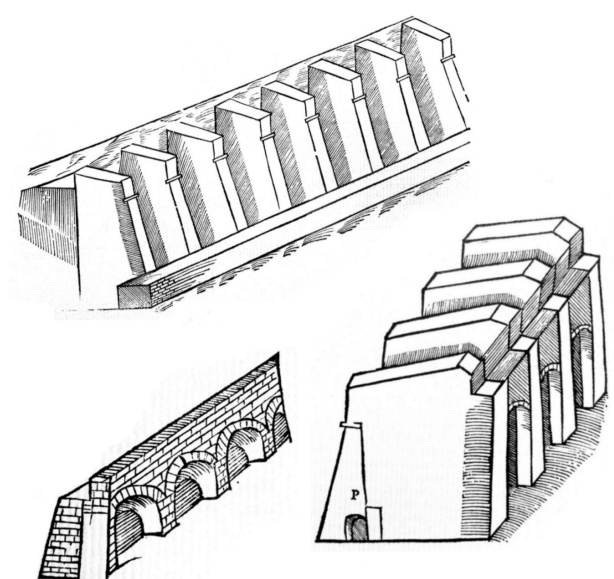

Fig. 7. Modelli grafici proposti nel trattato Della fortificatione delle città (Maggi, Castriotto, 1564) e riferimento progettuale per i fratelli Paleari Fratino (Casu, 2004).
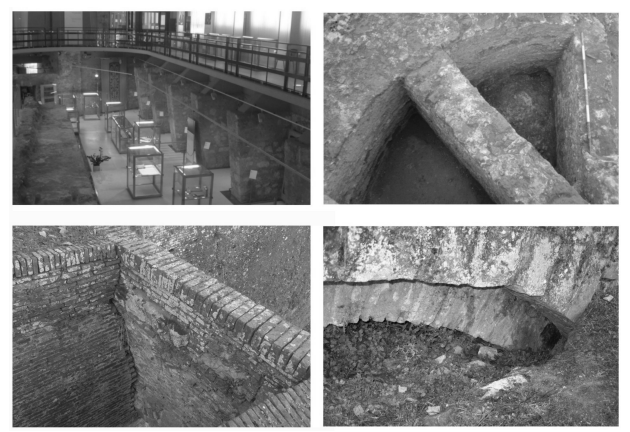

Fig. 6. Tecnica costruttiva impiegata a Cagliari, Alghero, Lucca (Italia) e Pamplona (Spagna).

La produzione di modelli grafici diviene in questa fase di studio la condizione necessaria per il progetto della successiva indagine geofisica mirata all'acquisizione di ulteriori informazioni utili ad avvalorare le ipotesi di partenza.

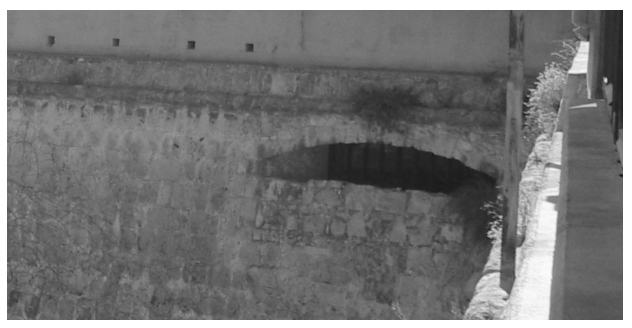

Fig. 8. Cannoniera realizzata dai Paleari nel periodo 1573-1578 al servizio della tenaglia di San Pancrazio.

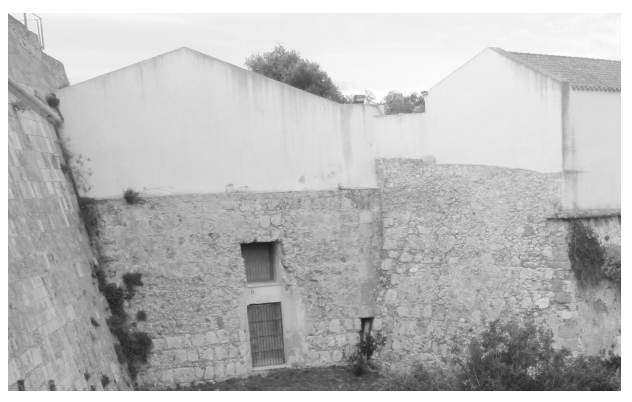

Fig. 9. Cannoniera nel fianco del bastione di Santa Croce posta in opera dai Paleari nel periodo 1573-1578.

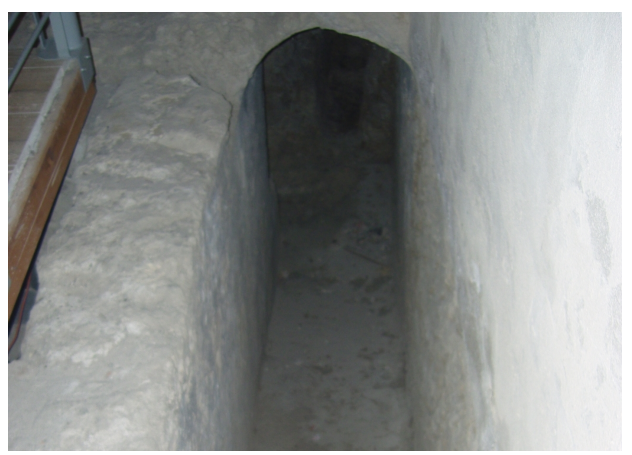

Fig. 10. Passaggio voltato al servizio del bastione di Santa Croce riconducibile all'opera dei fratelli Paleari.

\section{Rilievo e rappresentazione della cortina di Santa Chiara nel Cinquecento}

I documenti analizzati forniscono indicazioni sugli aspetti formali, dimensionali e costruttivi dell'opera cinquecentesca senza tuttavia descrivere gli spazi interni del sistema bastionato (co- 
me la cannoniera in casamatta) funzionali all'opera militare. Tuttavia è possibile, a partire da un rilievo aerofotogrammetrico del contesto urbano, definire un modello digitale dell'area studiata e -sulla base delle informazioni dedotte dall'analisi dei documenti archivistici, della trattatistica e delle opere realizzate dai Paleari- proporre alcune ipotesi ricostruttive (Figg. 12 e 16).

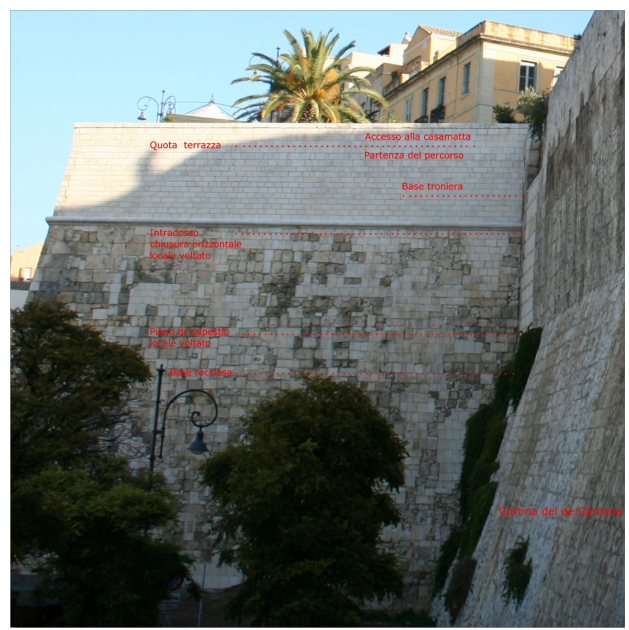

Fig. 11. Veduta del fianco della cortina di Santa Chiara con l'indicazione delle quote dell'opera militare note o ipotizzate.

La scelta della soluzione elaborata -che potrà trovare conferma o elementi di discussione a seguito dell'interpretazione dei dati offerti dalle indagini geofisiche- consiste in un terrapieno sostenuto da setti collegati da strutture voltate con un passaggio voltato che, dalla quota della piazza del nuovo baluardo, conduce alla cannoniera in casamatta. Questa postazione -integrata dalla artiglieria sistemata alla quota del cammino di ronda- vigila in direzione ovest verso il quartiere medievale di Stampace ed incrocia il tiro con la postazione presente nel fianco della cortina di Santa Chiara.
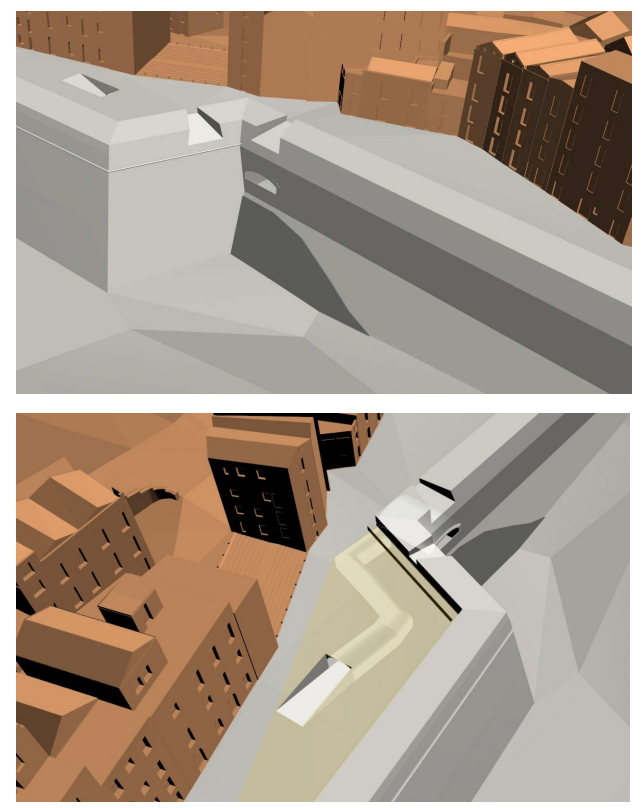

Fig. 12. Rappresentazione del settore in esame conforme al progetto del 1578. Si evidenzia l'accesso e il percorso di accesso alla cannoniera in casamatta posizionata a ridosso della cortina del de Cardona (15301540) e apertura per artiglieria conforme a quanto realizzato nella tenaglia di San Pancrazio (Fig. 8) nel periodo 1573-1578 (modello digitale a cura di M. Utzeri).

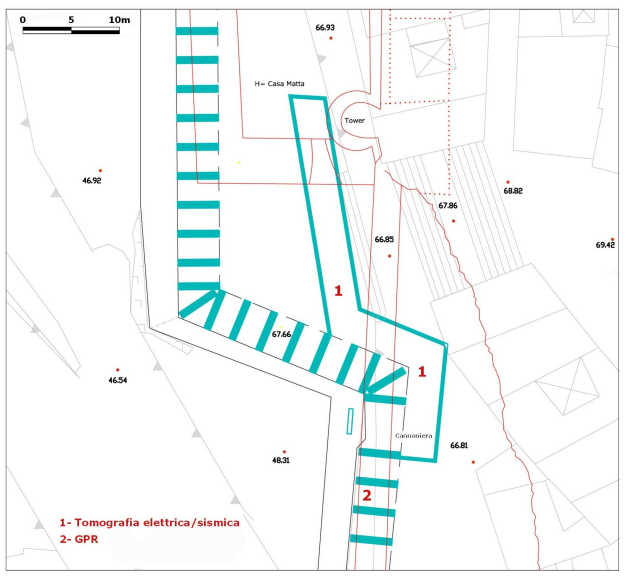

Fig. 13. Inserimento del disegno del 1575 su base attuale con ipotesi di accesso alla casamatta dotata di cannoniera e indicazione delle aree nelle quali è prevista l'indagine con metodi geofisici (rielaborazione grafica a cura di A. Pirinu). 


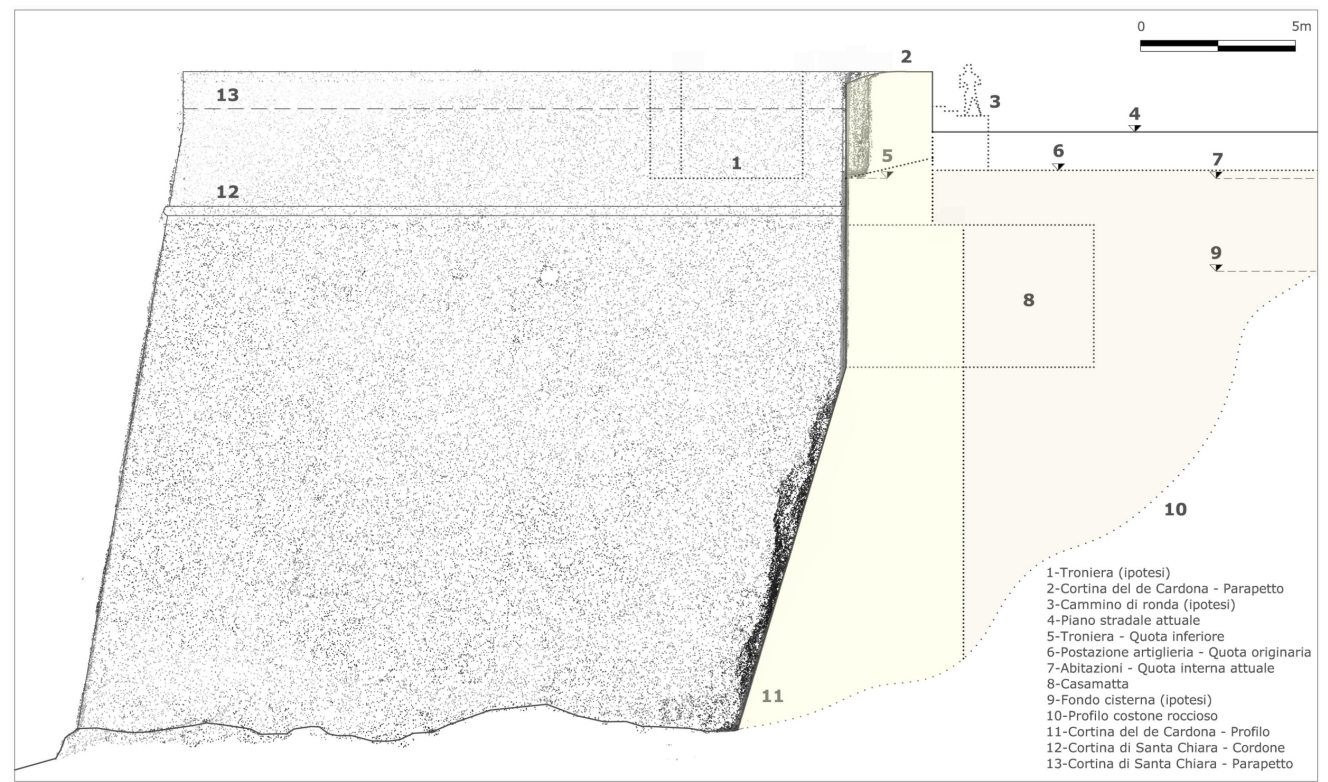

Fig. 14. Sezione prospetto realizzata a partire dal rilievo fotogrammetrico integrato con il rilievo strumentale che propone un'ipotesi di posizionamento della casamatta (rilievo e rappresentazione a cura di A. Pirinu, R. Argiolas).

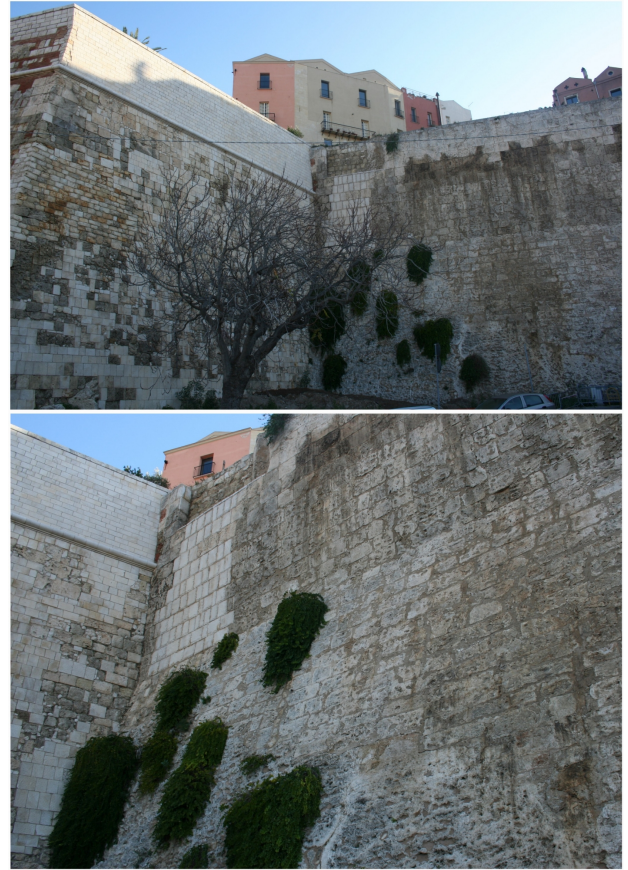

Fig. 15. Vedute del settore oggetto di studio interessato da restauri recenti del paramento murario.

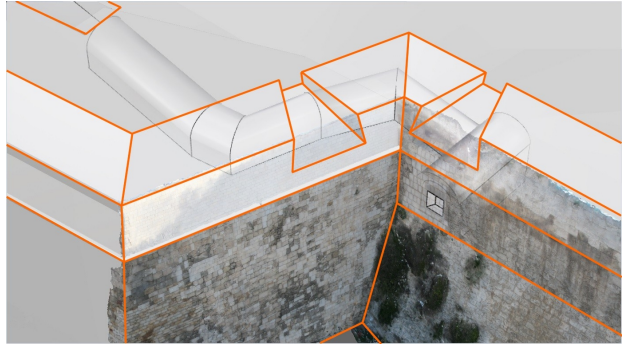

Fig. 16. Modello trimensionale che abbina lo stato attuale con un'ipotesi ricostruttiva del bastione cinquecentesco. L'apertura della cannoniera in casamatta viene proposta -per sagoma e dimensione- conforme a quanto realizzato dai Paleari nel fianco ritirato del bastione di Santa Croce (Fig. 9).

\section{Risultati raggiunti, loro applicabilità e pro- spettive di ricerca}

Il rilievo dell' aspetto esteriore dei bastioni cinquecenteschi unitamente all'osservazione della cartografia storica, allo studio della trattatistica militare ed all'analisi storico-archivistica ha condotto alla definizione di un primo modello digitale dell'opera militare, funzionale al proseguo delle indagini. Il database informativo così definito riceve le informazioni dedotte 
dall'analisi dei dati acquisiti e indirizza la scelta della successiva indagine geofisica.

Questo ulteriore passaggio permette difatti senza ricorrere a interventi invasivi non consentiti in un sito pluristratificato quale è l'ambito urbano in esame- di acquisire informazioni aggiuntive riferite al sistema costruttivo del bastione ed alla presenza di percorsi e spazi un tempo necessari per l'uso dell' architettura militare.

In questa fase la scelta metodo geofisico deriva dalla conoscenza di potenzialità e limiti offerti dai differenti sistemi d'indagine in particolare qualora si operi in ambito urbano.

L'ipotetica presenza di ambienti voltati o strutture murarie in generale posizionati a profondità ben definite (superiori a 3,00 m) rispetto all'attuale piano stradale (Fig. 14) escludono l'impiego estensivo del GPR al quale viene preferita la tomografia sismica (Figg. 18 e19) o la tomografia elettrica.

L'impiego del GPR tuttavia può trovare utile applicazione nella ricerca di strutture posizionate più a ridosso del piano d'azione dello strumento, come i sistemi costruttivi del bastione (Fig. 20) e le tracce di strutture abitative documentate nella prima metà del Cinquecento, che si ipotizza siano state inglobate -piuttosto che demolite- a seguito dell'avanzamento del fronte bastionato.

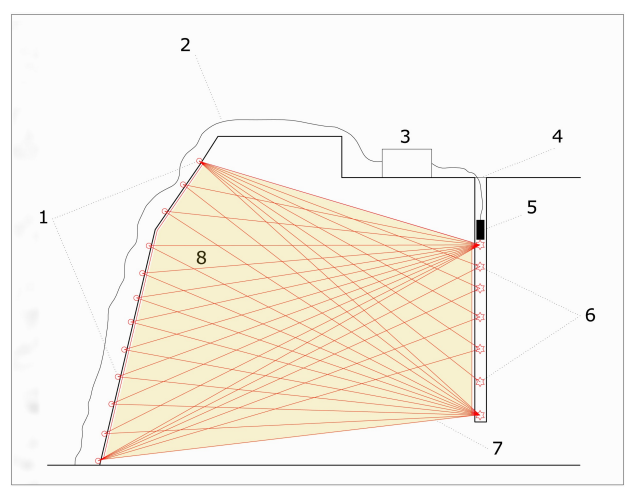

Fig. 17. Schema di tomografia uphole. 1) Geofoni; 2) Cavo dei geofoni; 3) Apparato acquisitore; 4) Foro di sondaggio; 5) Sorgente di energia; 6) Energizzazioni; 7) Raggi sismici. (Schema grafico da Pirinu, Balia, 2018).
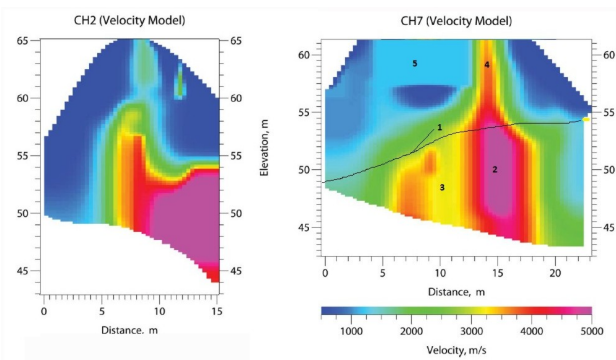

Fig. 18. Applicazione di tomografia sismica lungo la faccia ovest del bastione di Santa Croce (Balia, Pirinu, 2018). Le immagini mostrano con chiarezza la base rocciosa, il corpo murario riconducibile alle strutture del bastione ed il riempimento costituito dal terrapieno.

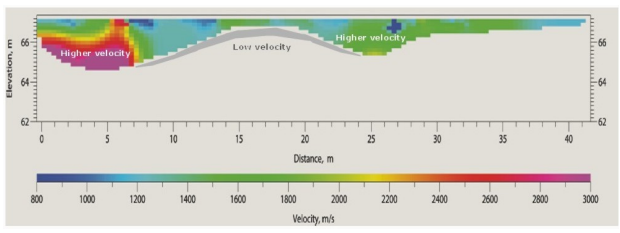

Fig. 19. Applicazione di tomografia a rifrazione lungo un tratto della cortina di Santa Chiara interessato a partire dalla fine del Cinquecento da crolli e ricostruzioni storiche documentate ed alla fine degli anni '90 del Novecento oggetto di interventi di manutenzione straordinaria che hanno previsto l'impiego di pali in cls armato (Pirinu, et al., 2018).

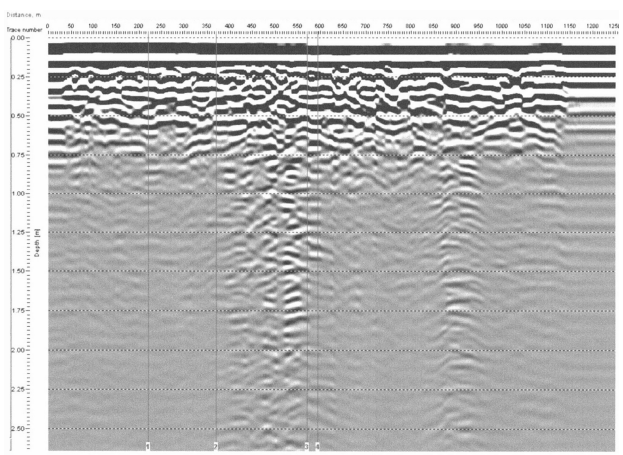

Fig. 20. Indagine con GPR all'interno delle abitazioni presenti lungo il perimetro ovest del bastione di Santa Croce a Cagliari. Si distingue chiaramene la sagoma dei setti in muratura (spessore $60-70 \mathrm{~cm}$ ) a sostegno del terrapieno, leggibile - una volta superato il disturbo dovuto alla chiusura di base costituita da granchi in pvc e massetto in cls con rete elettrosaldata- sino a m 2,50 al di sotto del piano di calpestio (schema grafico da Pirinu, Balia, 2018). 


\section{Bibliography}

Balia, R.; Pirinu, A. (2018). "Geophysical surveying of the ancient walls of the town of Cagliari, Italy, by means of refraction and uphole seismictomography techniques", Archaeological Prospection, 25(2), pp. 1-7.

Cadinu, M.; Pirinu, A.; Schirru, M. (2013). "Letture catastali, rilievi, e documenti per la lettura delle architetture e dell'urbanistica dell'area di Santa Croce del Castello di Cagliari”, in Cadinu, M. ed., I Catasti e la storia dei luoghi, Edizioni Kappa, Roma, pp. 509-541.

Casu, S. (2002). "Cagliari, un secolo di restauro delle fortificazioni", in Atti del convegno internazionale Castelli in terra, in acqua e... in aria, Alinea, Firenze, pp. 212-218.

Maggi, G.; Castriotto, G. (1982). Della fortificatione delle città di M. Girolamo Maggi e del Capitan Iacomo Castriotto (1583), Jouvence Viella Ed., Roma.

Mele, M.G. (2019). "Storia e realtà insediativa della città di Cagliari nella prima metà del XVI secolo", in Mele, M.G.R., ed., Mediterraneo e città. Discipline a confronto, Franco Angeli Editore, Milano, pp. 53-69.

Milanese, M. (2012). “Archeologia delle piazzaforti spagnole della Sardegna nord-occidentale (Alghero, Bosa e Castelsardo)”, Archeologia Postmedievale, 13/2009, All'insegna del Giglio, Firenze, pp. 141-170.

Pirinu, A. (2013). Il disegno dei baluardi cinquecenteschi nell'opera dei fratelli Paleari Fratino. Le piazzeforti della Sardegna, All'insegna del Giglio, Firenze.

Pirinu, A.; Balia R. (2018). "Riconoscimento e tutela di un complesso monumentale storico in ambiente urbano mediante l'integrazione di metodologie di rilevamento e di tecniche geofisiche non distruttive: l'area di Santa Croce nel quartiere Castello a Cagliari”, Restauro Archeologico, Dipartimento di Architettura, Firenze, pp. 74-89.

Pirinu, A.; Balia, R.; Piroddi, L.; Trogu, A.; Utzeri, M.; Vignoli, G. (2018). "Deepening the knowledge of military architecture in an urban context through digital representations integrated with geophysical surveys", The city walls of Cagliari (Italy), in atti del 2018 IEEE International Conference on Metrology for Archaeology and Cultural Heritage, Cassino, pp. 206-210.

Rassu, M. (2003). Baluardi di pietra, Aipsa Edizioni, Ortacesus.

Schirru, M. (2017). Le residenze signorili nella Sardegna moderna, Carlo Delfino Ed., Sassari.

Viganò, M. (2004). El fratin mi yngeniero, I Paleari Fratino da Morcote ingegneri militari ticinesi in Spagna (XVIXVII secolo), Istituto grafico Casagrande SA, Bellinzona. 